\title{
Transgenic rabbits with increased VEGF expression develop hemangiomas in the liver: a new model for Kasabach-Merritt syndrome
}

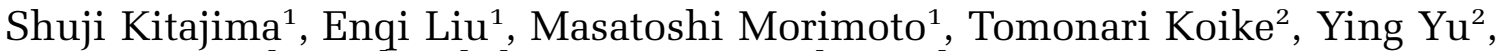 \\ Teruo Watanabe ${ }^{3}$, Shigehiko Imagawa ${ }^{4}$ and Jianglin Fan $^{2}$ \\ ${ }^{1}$ Analytical Research Center for Experimental Sciences, Saga University, Saga, Japan; ${ }^{2}$ Cardiovascular \\ Disease Laboratory, Department of Pathology, Institute of Basic Medical Sciences, University of Tsukuba, \\ Tsukuba, Japan; ${ }^{3}$ President's Office, Saga University, Saga, Japan and ${ }^{4}$ Division of Hematology, Institute \\ of Clinical Medicine, University of Tsukuba, Tsukuba, Japan
}

\begin{abstract}
Clinical studies have provided ample evidence that high (either systemic or local) levels of vascular endothelial growth factor (VEGF) are associated with several pathophysiological disorders, including hemangiomas. To investigate whether elevated VEGF expression could directly affect these disorders, we created a transgenic $(\mathrm{Tg})$ rabbit model with increased hepatic expression of the human VEGF $_{165}$ transgene under the control of the human $\alpha$-antitrypsin promoter. Tg rabbits exhibited marked hepatomegaly, with livers 2.5-fold heavier than those of control rabbits. Histological analysis revealed that the livers of $\mathrm{Tg}$ rabbits showed prominent dilation of the sinusoids and formed various-sized blood vessel networks, a feature of diffuse hemangiomas. Immunohistochemical staining revealed that the hepatocytes produced VEGF $_{165}$, whereas plasma VEGF We5 $_{165}$ not detected. Furthermore, Tg rabbits suffered from hemolytic anemia, thrombocytopenia and splenomegaly, which was associated with marked extramedullary hematopoiesis. The manifestations of Tg rabbits mimic many of the features of hemangiomatous disorders in humans such as the Kasabach-Merritt syndrome, and therefore this model may be potentially useful for the study of the pathogenesis and complications of hemangiomas as well as the investigation of angiogenesis inhibitors.
\end{abstract}

Laboratory Investigation (2005) 85, 1517-1527. doi:10.1038/labinvest.3700346; published online 3 October 2005

Keywords: anemia; hemangioma; Kasabach-Merritt syndrome; transgenic rabbit; VEGF

Abnormal angiogenesis is involved in many pathophysiological processes. Vascular endothelial growth factor (VEGF), also known as vascular permeability factor, is one of the most important endothelial cell (EC)-specific mitogen and angiogenesis inducer. ${ }^{1}$ VEGF mRNA undergoes alternative splicing events that generate four different homodimeric glycoproteins designated as $\mathrm{VEGF}_{121}$, $\mathrm{VEGF}_{165}, \mathrm{VEGF}_{189}$ and $\mathrm{VEGF}_{206}$. VEGF 121 is a nonheparin-binding acidic protein, which is freely diffusible, whereas the longer forms, $\mathrm{VEGF}_{189}$ or VEGF $_{206}$, are highly basic proteins tightly bound to extracellular heparin-containing proteoglycans. However, $\mathrm{VEGF}_{165}$, which has intermediate properties, is the predominant isoform and has potent biological activity. ${ }^{1,2}$ The biological effects of VEGF

Correspondence: Dr J Fan, MD, PhD, Cardiovascular Disease Laboratory, Department of Pathology, Institute of Basic Medical Sciences, University of Tsukuba, Tsukuba 305-8575, Japan.

E-mail: J-LFAN@md.tsukuba.ac.jp

Received 17 April 2005; revised 3 August 2005; accepted 4

August 2005; published online 3 October 2005 are mediated by two tyrosine kinase receptors, VEGFR-1 and VEGFR-2.,

VEGF is essential for embryogenesis, wound healing and corpus luteum formation and even deletion of a single allele of the VEGF gene results in embryonic lethality in VEGF-deficient mice. ${ }^{3,4}$ Accumulating evidence has revealed that VEGF plays important roles in pathophysiological conditions with neovascularization, and that upregulation of VEGF expression may be associated with hemangiomas, ${ }^{5,6}$ diabetic retinopathy ${ }^{7}$ and nephropathy, ${ }^{8,9}$ atherosclerosis ${ }^{10-12}$ and rheumatoid arthritis. ${ }^{13}$ In addition, VEGF is involved in the proliferation of several types of tumor cells and the administration of monoclonal antibodies (mAbs) against VEGF suppressed the growth of human tumors implanted into nude mice. ${ }^{14}$ In diabetic retinopathy, hypoxia present in peripheral vessels of diabetic patients may induce local expression of VEGF in the retina, which results in abnormal angiogenesis and subsequently leads to retinal detachment, hemorrhage and blindness. One study demonstrated that overexpression of VEGF in the retina of transgenic (Tg) 
mice is sufficient to cause intraretinal and subretinal neovascularization. ${ }^{15}$ On the other hand, clinical studies also revealed that high plasma levels of VEGF were associated with rheumatoid arthritis, ${ }^{13}$ hemangioma $^{5,6}$ and anemia. ${ }^{16}$ However, it is unknown whether the increased VEGF in the plasma in these patients is causative or a consequence, or both of these disorders.

These lines of evidence prompted us to generate a Tg rabbit model to study the specific pathological consequences of chronic VEGF overexpression in the liver or/and blood in vivo. Rabbits were selected for this undertaking because of their usefulness in the study of atherosclerosis, ${ }^{17}$ diabetes mellitus ${ }^{18}$ and arthritis, ${ }^{19}$ and also because our laboratory has been using Tg rabbits to investigate these diseases. In addition, the large size of the eyeballs of rabbits may facilitate retinal studies, such as studies of diabetic retinopathy. To generate $\mathrm{Tg}$ rabbits with high levels of VEGF, we chose to use the $\mathrm{VEGF}_{165}$ isoform under the control of a hepatic-specific promoter ( $\alpha$-antitrypsin promoter). Previous studies using cultured cells showed that at least a fraction of VEGF $_{165}$ may be released from the cellular surface. ${ }^{20}$ We envisioned that hepatic overexpression of VEGF $_{165}$ in Tg rabbits may result in two possible pathological events: (1) increased local hepatic expression of $\mathrm{VEGF}_{165}$ may cause vascular malformations in the livers of Tg rabbits or (2) Tg rabbits may have elevated plasma levels of $\mathrm{VEGF}_{165}$, so that we could possibly investigate the cause-and-effect relationship between VEGF and pathological states such as atherosclerosis. In this study, we characterized $\mathrm{Tg}$ rabbits and demonstrated that hepatically expressed $\mathrm{VEGF}_{165}$ was exclusively bound to the extracellular matrix, and thereby directly induced hemangiomatosis. Furthermore, these $\mathrm{Tg}$ rabbits developed many of the features of the KasabachMerritt syndrome, including thrombocytopenia, anemia and splenomegaly.

\section{Materials and methods}

\section{Generation of Tg Rabbits}

Tg rabbits were generated by the methods described previously. ${ }^{17}$ In this study, Japanese White (JW:kbt) rabbits (Biotek Co., Saga, Japan) were used and zygotes were injected with a DNA construct consisting of human $\mathrm{VEGF}_{165}$ cDNA (Genbank No. NM_003376) under the control of a hepatic promoter, the human $\alpha$-antitrypsin promoter (Figure 1). ${ }^{21}$ Rabbits were housed individually in metal cages in a room maintained at constant temperature $\left(24 \pm 2^{\circ} \mathrm{C}\right)$ and humidity $(55 \pm 15 \%)$. The experimental protocol and design were approved by the Saga University Animal Experimentation Committee and performed according to the Saga University Guidelines for Animal Experimentation. The presence of the transgene in offspring was examined by Southern blot analysis. Hybridization was performed with a ${ }^{32}$ P-labeled hVEGF cDNA probe synthesized by using a Prime-It II random primer labeling kit. ${ }^{22}$

\section{Northern Blotting and Reverse Transcriptase-Polymerase Chain Reaction Analysis}

Total RNA from various tissues was rapidly isolated using Trizol reagent (Invitrogen, CA, USA). RNA $(10 \mu \mathrm{g})$ was then subjected to electrophoresis in a $1.2 \%$ agarose gel and transferred to a Nytran nylon membrane. The membrane was hybridized in turn with the ${ }^{32}$ P-labeled hVEGF cDNA probe described above and then rehybridized with a $\beta$-actin cDNA probe as an internal standard. Total RNA was reverse-transcriptase into cDNA by using Invitrogen reverse transcription reagents and real-time reverse transcriptase-polymerase chain reaction (RT-PCR) (DNA Engine Option; MJ Research, Tokyo, Japan) was performed using a DyNAmo SYBR Green qPCR kit (Finnzymes) according to the manufacturer's instructions. ${ }^{19}$ The specific primer for hVEGF was: $5^{\prime}$-GGC AGA ATC ATC ACG AAG-3' and 5'-CAG GAT GGC TTG AAG ATG-3'.

\section{Histological Examinations and Immunohistochemistry}

For morphological analysis, Tg and littermate control rabbits were killed and the liver, kidney, spleen, heart, lung, pancreas and bone marrow were removed and fixed in a $10 \%$ neutral buffed formalin solution. These tissues were embedded in paraffin and cut in 4- $\mu \mathrm{m}$ sections and stained with hematoxylin and eosin (H-E) and Elastic van Gieson (EVG)

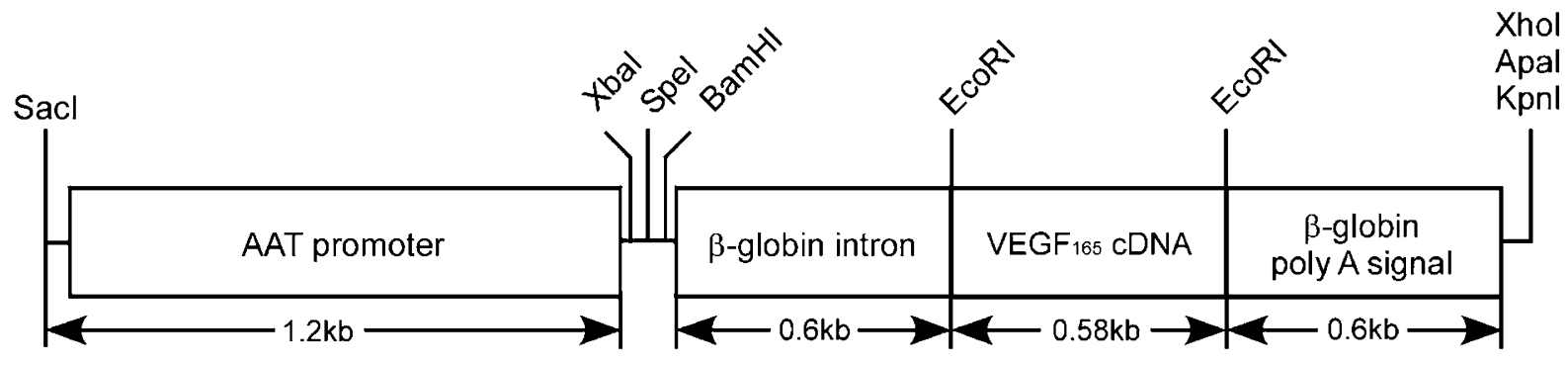

Figure 1 Schematic illustration of the Tg construct. An AAT-hVEGF ${ }_{165}$ construct containing the human $\alpha 1$-antitrypsin (AAT) promoter, rabbit $\beta$-globin intron and rabbit $\beta$-globin poly A signal was used for microinjection. 
for light microscopic examination. MAbs against human VEGF (1:100) (NeoMarkers, CA, USA), $\alpha$ smooth muscle actin ( $\alpha$-SMA) (1:200) (Dako Corporation, Carpinteria, CA, USA) and CD31 (1:100) (Dako) were used. Immunohistochemistry was performed with the Dako EnVision system following the manufacturer's instructions.

\section{Electron Microscopy}

A small piece of liver was fixed in $2.5 \%$ glutaraldehyde and postfixed in $1 \%$ osmium tetraoxide using a standard protocol. Ultrathin sections were doubly stained with uranyl acetate and lead citrate, and were observed under an JEM-100CX electron microscope as described previously. ${ }^{23}$

\section{Hematological Analysis}

Blood was collected from control and Tg rabbits at the ages of 14 and 20 weeks. The number of red blood cells (RBCs), white blood cells (WBCs) and platelets was counted using an automated hematology analyzer Sysmex K-4500 (Sysmex Co., Kobe, Japan) and hematocrits (Ht) were determined with capillary tubes centrifuged at 10000 r.p.m. for $5 \mathrm{~min}$. The number of reticulocytes was counted by flow cytometry using an automated reticulocyte analyzer Sysmex R-3500 (Sysmex). Hemoglobin (Hb) contents were measured using $\mathrm{Hb}$ analysis kits (Wako Purechemical, Osaka, Japan). Mean corpuscular volume (MCV), mean corpuscular hemoglobin $(\mathrm{MCH})$ and mean corpuscular hemoglobin concentration (MCHC) were calculated and are shown in Table 1. The plasma iron and total iron binding capacity (TIBC) were measured by the nitorosoPSAP method using an autoanalyzer Hitachi 7170 (Hitachi High-Technologies Co., Tokyo, Japan). Smear samples of peripheral blood were examined

Table 1 Hematological analysis of VEGF transgenic rabbits

\begin{tabular}{lcc}
\hline & Cont $(\mathrm{n}=6)$ & Transgenic $(\mathrm{n}=6)$ \\
\hline RBC $\left(10^{4} / \mu \mathrm{l}\right)$ & $646.5 \pm 43.4$ & $391.0 \pm 34.8^{* *}$ \\
WBC $\left(10^{2} / \mu \mathrm{l}\right)$ & $88.5 \pm 6.1$ & $92.8 \pm 5.8$ \\
Platelet $\left(10^{4} / \mu \mathrm{l}\right)$ & $46.9 \pm 4.0$ & $21.2 \pm 4.6^{* *}$ \\
Reticulocyte $(\%)$ & $23.3 \pm 1.0$ & $65.3 \pm 5.5^{* *}$ \\
$\mathrm{Ht}(\%)$ & $44.7 \pm 2.8$ & $26.7 \pm 1.3^{* *}$ \\
$\mathrm{Hb}(\mathrm{g} / \mathrm{dl})$ & $14.5 \pm 0.9$ & $7.8 \pm 0.3^{* *}$ \\
$\mathrm{MCV}\left(\mu^{3}\right)$ & $69.2 \pm 0.8$ & $65.7 \pm 1.4^{*}$ \\
$\mathrm{MCH}(\mathrm{pg})$ & $22.2 \pm 0.3$ & $19.3 \pm 0.8^{*}$ \\
$\mathrm{MCHC}(\%)$ & $32.7 \pm 0.2$ & $29.5 \pm 0.6^{* *}$ \\
$\mathrm{Fe}(\mu \mathrm{g} / \mathrm{dl})$ & $196.3 \pm 26.5$ & $72.5 \pm 0.6^{* *}$ \\
TIBC $(\mu \mathrm{g} / \mathrm{dl})$ & $280.5 \pm 22.0$ & $318.2 \pm 23.0$ \\
\hline
\end{tabular}

Data are expressed as mean \pm s.e.m.

RBC, red blood cell; WBC, white blood cell; MCV, mean corpuscular volume $(\mathrm{MCV}=\mathrm{Ht} / \mathrm{RBC} \times 1000) ; \mathrm{MCH}$, mean corpuscular hemoglobin $(\mathrm{MCV}=\mathrm{Hb} / \mathrm{RBC} \times 1000)$; MCHC, mean corpuscular hemoglobin concentration $(\mathrm{MHCH}=\mathrm{Hb} / \mathrm{Ht} \times 100)$; TIBC, total iron binding capacity. ${ }^{*} P<0.05,{ }^{*} P<0.01$ vs controls. after Giemsa staining. Abnormalities of RBCs in Tg rabbits were investigated under a scanning electron microscope. ${ }^{24}$ The cellularity of bone marrow was determined using the Lumina-Vision image analysis system (Mitani Co., Tokyo, Japan) and megakaryocytes were calculated by counting 10 high-power field (HPF) at random.

\section{Surgical Removal of the Hepatic Hemangiomas}

To investigate whether the surgical removal of the hepatic hemangiomas was able to improve thrombocytopenia and anemia in Tg rabbits (see below), we excised $\sim 50 \%$ of the liver (with gross lesions) of $\mathrm{Tg}$ rabbits at age of 6 months using the protocol described by Yamaoka et $a l^{25}$ and monitored the changes of blood platelets and RBC count.

\section{Statistical Analysis}

Results were expressed as the mean \pm s.e.m. Statistical analysis was performed using either the Student's $t$-test when the $F$-value was equal or Welch's $t$-test when the $F$-value was not equal. $P<0.05$ was considered statistically significant.

\section{Results}

A total of 1804 zygotes were microinjected and 137 pups were obtained. Among them, six pups were found by Southern blot analysis to have the hVEGF transgene integration. Two live founder Tg rabbits (designated V58 and V112) were mated with non-Tg rabbits to create and expand colonies. However, V58 Tg rabbits did not show detectable hVEGF RNA expression in the liver and hepatic phenotype; therefore, we used V112 F1 Tg rabbits for the current study. Tg rabbits showed no obvious abnormal phenotype and appeared healthy except for slightly lower body weight compared with control littermates ( $n=6$ for each group) after 12 weeks of age, but the difference was not statistically significant. Analysis of plasma by two hVEGF-specific ELISA kits (Genzyme Techne Co., MN, USA and Pierce Endogen, IL, USA) did not detect hVEGF (all values were below the detection limit) in $\mathrm{Tg}$ rabbits, suggesting that $\mathrm{hVEGF}_{165}$ was not released into the circulation.

\section{Northern Blotting and RT-PCR Analysis}

Northern blotting analysis revealed that rabbit endogenous VEGF was ubiquitously expressed in multiple tissues (Figure 2a). Specific expression of hVEGF $_{165}$ (smaller than rabbit endogenous VEGF) was detected only in the liver by Northern blotting analysis, and the specificity of hepatic expression was confirmed by real-time RT-PCR analysis using hVEGF-specific primers (Figure 2b). hVEGF pro- 

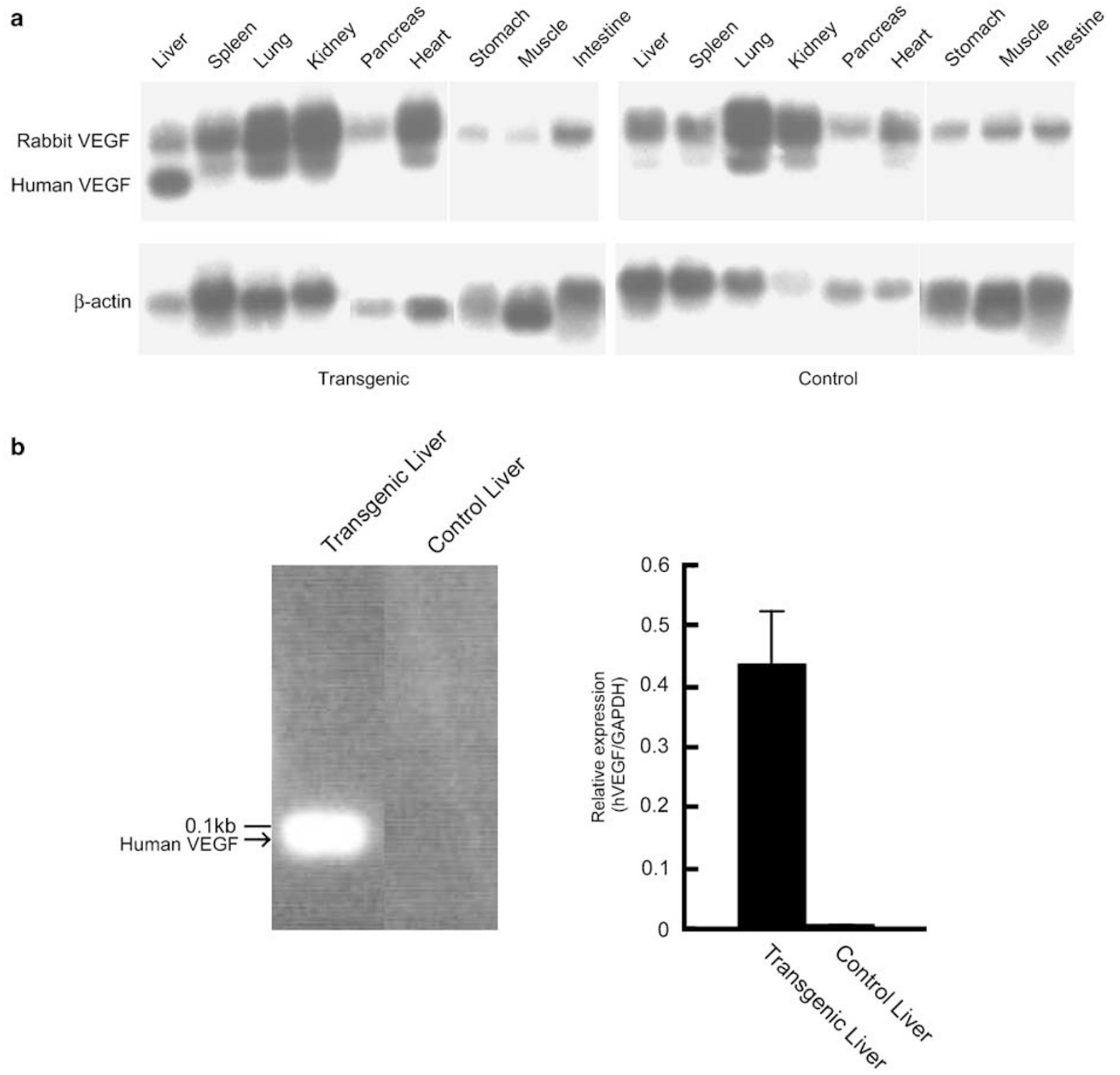

Figure 2 Northern blotting analysis (a) and RT-PCR (b) of VEGF mRNA tissue distribution in Tg rabbits. Rehybridization of the membrane with a $\beta$-actin probe showed that similar amounts of RNA had been loaded in each lane. Human VEGF expression was found only in Tg rabbit liver. The specific expression of hVEGF was confirmed by real-time RT-PCR (b).

teins were produced by hepatocytes in Tg rabbits, as confirmed by immunohistochemical staining (Figure 3).

\section{Pathological Examinations}

Liver

Rabbits from V112 Tg line were killed and examined at 8,16 and 24 weeks of age. The most pronounced gross finding in $\mathrm{Tg}$ rabbits was that $\mathrm{Tg}$ rabbits had enlarged livers and spleens compared to control rabbits (Figure 4). These changes were not prominent in young rabbits (8 weeks) but became visible in old rabbits. The average weight of the livers in Tg rabbits
(24 weeks) was 2.5 -fold heavier than that in control rabbits $(80.2 \pm 7.2 \mathrm{~g}$ in control vs $199.4 \pm 22.7 \mathrm{~g}$ in $\mathrm{Tg}$, $n=5$ for each group, $P<0.01$ ) (Figure $4 \mathrm{a}$ ). The surface of the liver was rough, and it consisted of deep-red regions divided by whitish areas. The cut surface after fixation showed many dilated vessels separated by whitish spongy structures (Figure 4b).

Histological analysis revealed several pathological changes in the livers of Tg rabbits. First, there were numerous foci composing a melange of irregular and various-sized vascular channels, including venous, cavernous and capillary-size vessels (Figure 5b, c). These malformed vessels were haphazardly scattered throughout the whole liver. They were tortuous and formed various-sized spaces filled with 


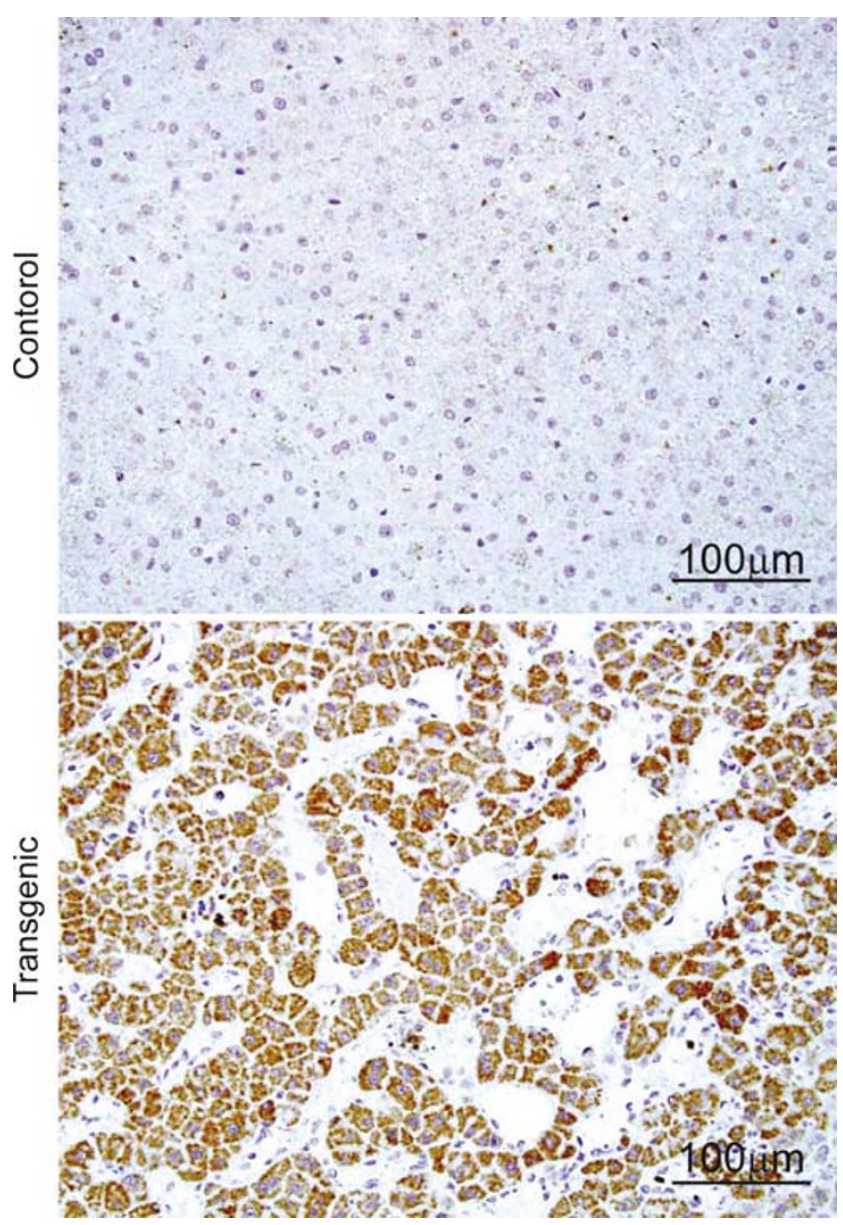

Figure 3 Immunohistochemical demonstration of hVEGF ${ }_{165}$ protein in the liver of a Tg rabbit. Note that hVEGF $_{165}$ was expressed by hepatocytes.

erythrocytes. They were separated by relatively scanty fibrous stroma in which capillaries or trapped hepatocytes were present. The lumen was lined by a layer of flattened ECs, beneath which some spindle-shaped cells (presumably smooth muscle cells, SMCs) were scattered (see below). The liver plates, with dilated sinusoids and vessels, were frequently hyperemic, suggesting that these vessels contained a large amount of blood. The lesions were not clearly demarcated; therefore, their features were compatible with those of hepatic angiomatosis or diffuse hemangioma ${ }^{26}$ (Figure 5b, c). The second striking change in Tg rabbit livers was the marked dilation of sinusoids of the liver plates and they are connected with the central veins (Figure $5 \mathrm{~d}$ ). Dilated sinuses were also lined by fattened ECs, beneath which $\alpha$-SMA-positive cells were present (presumably SMCs or pericytes) but they lacked elastin fibers (Figure 5d). Apart from these vascular channels and dilated sinuses, the hepatocytes showed focal degenerative changes, and many hepatocytes had dropped out or disappeared from the liver plates, and were replaced by amorphorous matrix or excessive edema (Figure 5e). In addition, there was marked extramedullary hematopoiesis in the liver. In such areas, foci of erythroid and myeloid precursors along with megakaryocytes were dispersed (Figure 5f). The nature of the lining ECs and SMCs was further investigated by immunohistochemical staining and electron microscopic observation. As shown in Figure 6, the lining ECs were positively stained by mAb CD31 and contained Weibel-Palade bodies in the cytoplasm, whereas SMCs were positive for $\alpha$-SMA.

\section{Spleen}

The spleens of $\mathrm{Tg}$ rabbits were larger and heavier than those of control rabbits $(1.1 \pm 0.1 \mathrm{~g}$ in control $v s$ $4.8 \pm 2 \mathrm{~g}$ in Tg, $n=5$ for each group, $P<0.01$ ) (Figure 4c). Histological analysis showed that the enlarged spleen of Tg rabbits was characterized by prominent extramedullary hematopoiesis along with accumulation of hemosiderin-containing macrophages (Figure 7, upper panel).

\section{Bone marrow}

Analysis of the bone marrow showed that Tg rabbits had hypercellularity $(32.8 \pm 6.2 \%$ in control vs $53.5 \pm 6.3 \%$ in Tg, $n=4$ for each group, $P<0.01$ ) (Figure 7, middle panel). Number of megakaryocytes of Tg rabbits was significantly increased compared to control rabbits: $4-11(5.5 \pm 2.3 / \mathrm{HPF}$ in $\mathrm{Tg})$ vs $0-3$ $(1.3 \pm 1.0 / \mathrm{HPF}$ in control).

\section{Hematological Analysis}

Since Tg rabbits exhibited the prominent extramedullary hematopoiesis in the liver and spleen, we further performed hematological analysis. Tg rabbits showed a significant decrease of $\mathrm{RBC}, \mathrm{Ht}, \mathrm{Hb}$ and platelets compared to control rabbits, whereas the WBC number was not changed (Table 1). The MC, MCH and MCHC in Tg rabbits were significantly lower than those in control rabbits (Table 1). These hematological findings along with the hypercellularity of bone marrow suggested that Tg rabbits suffered from microcytic hypochromic anemia. The number of reticulocytes in $\mathrm{Tg}$ rabbits was significantly increased compared with that in control rabbits (Table 1). Plasma iron levels in Tg rabbits were significantly lower than those in control rabbits (Table 1). There were no differences from control rabbits in glucose, total protein, blood urea nitrogen, creatinin, albumin, glutamic oxaloacetic transaminase (GOT) and glutamic pyruvic transaminase (GPT) (data not shown). Examinations of peripheral blood revealed that there were many deformed fragmented erythrocytes (Figure 7, bottom panel).

To explore whether hepatic hemangiomatous lesions were responsible for blood abnormalities such as thrombocytopenia in $\mathrm{Tg}$ rabbits, we performed partial hepatectomy and examined the changes of platelets and RBCs after surgery. As 

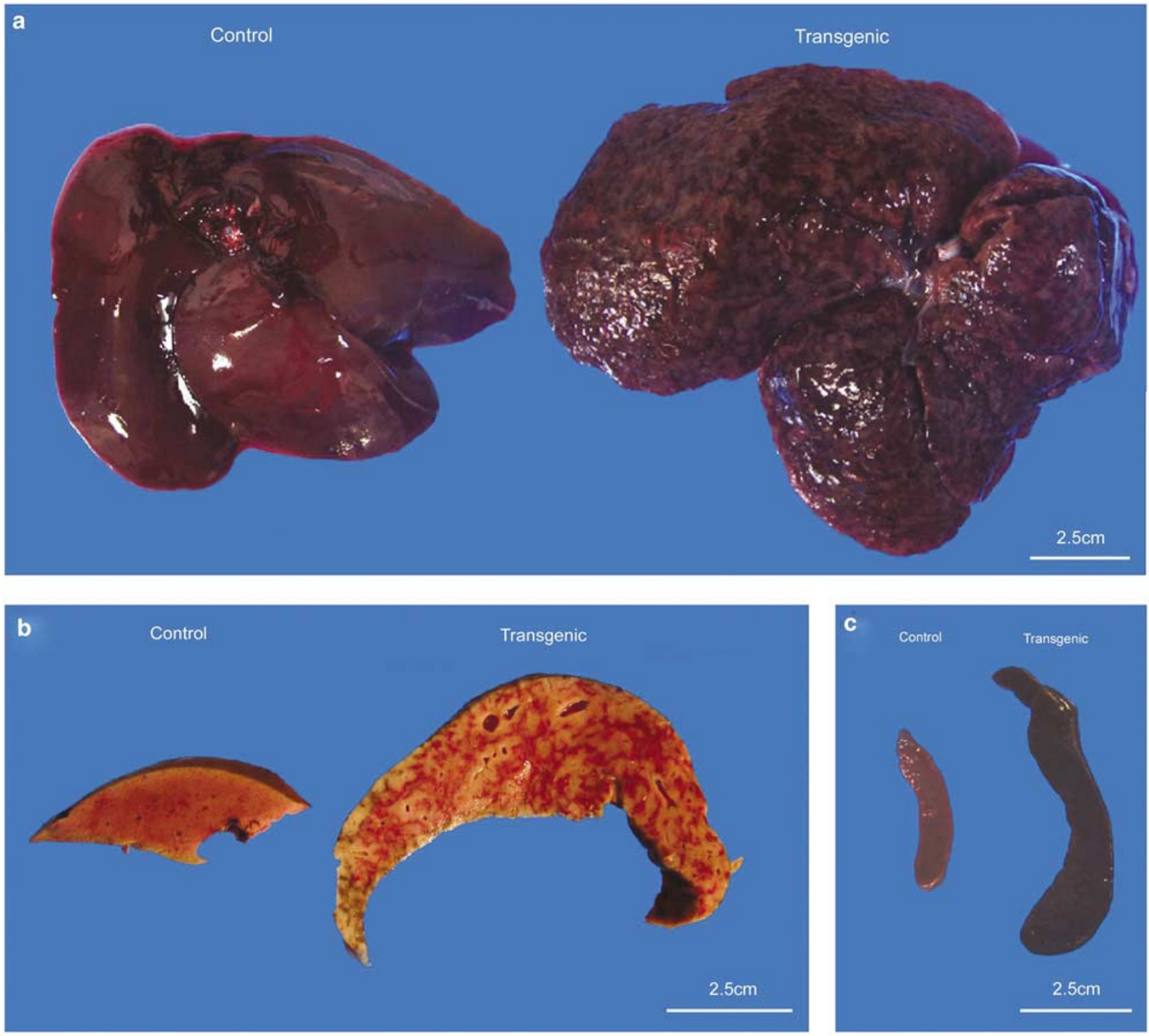

Figure 4 Macroscopic appearance of the liver and spleen. The weight of the livers of Tg rabbits was increased up to 3 times (a). After formalin fixation, some spongiform lesions were noticed on the cut surface of the liver (b). The spleen was also larger in Tg rabbits than in control rabbits (c).

shown in Figure 8, the surgical removal of the liver lesions corrected the number of platelets of $\mathrm{Tg}$ rabbits to the normal range while RBCs were not significantly improved.

\section{Discussion}

Several lines of evidence have shown that VEGF is upregulated in the hemangiomatous tissues of humans. ${ }^{6,27}$ To examine whether VEGF alone directly causes the formation of hemangiomas, we generated and characterized Tg rabbits that overexpressed human $\mathrm{VEGF}_{165}$ in the liver. We did not detect any $\mathrm{hVEGF}_{165}$ in the plasma, suggesting that $\mathrm{VEGF}_{165}$, like other longer $\mathrm{VEGF}_{189,202}$ isoforms, tightly binds to the extracellular matrix and functions locally.

Immunohistochemical analysis revealed that VEGF $_{165}$ protein was expressed by hepatocytes and possibly associated with heparin sulfate proteoglycans, which are abundantly present on the hepatocytes in the space of Disse and sinusoid ECs. Increased local expression of $\mathrm{VEGF}_{165}$ resulted in several pathological changes in the liver. The hepatic sinusoids were markedly dilated and many neovascular networks, including venous or cavernous vessels or capillaries, were formed, constituting the basis of the enlarged liver size in Tg rabbits. The lining ECs along the expanded sinusoid and deformed vessels were positive for CD31 mAb and contained Weibel-Palade bodies, suggesting that 

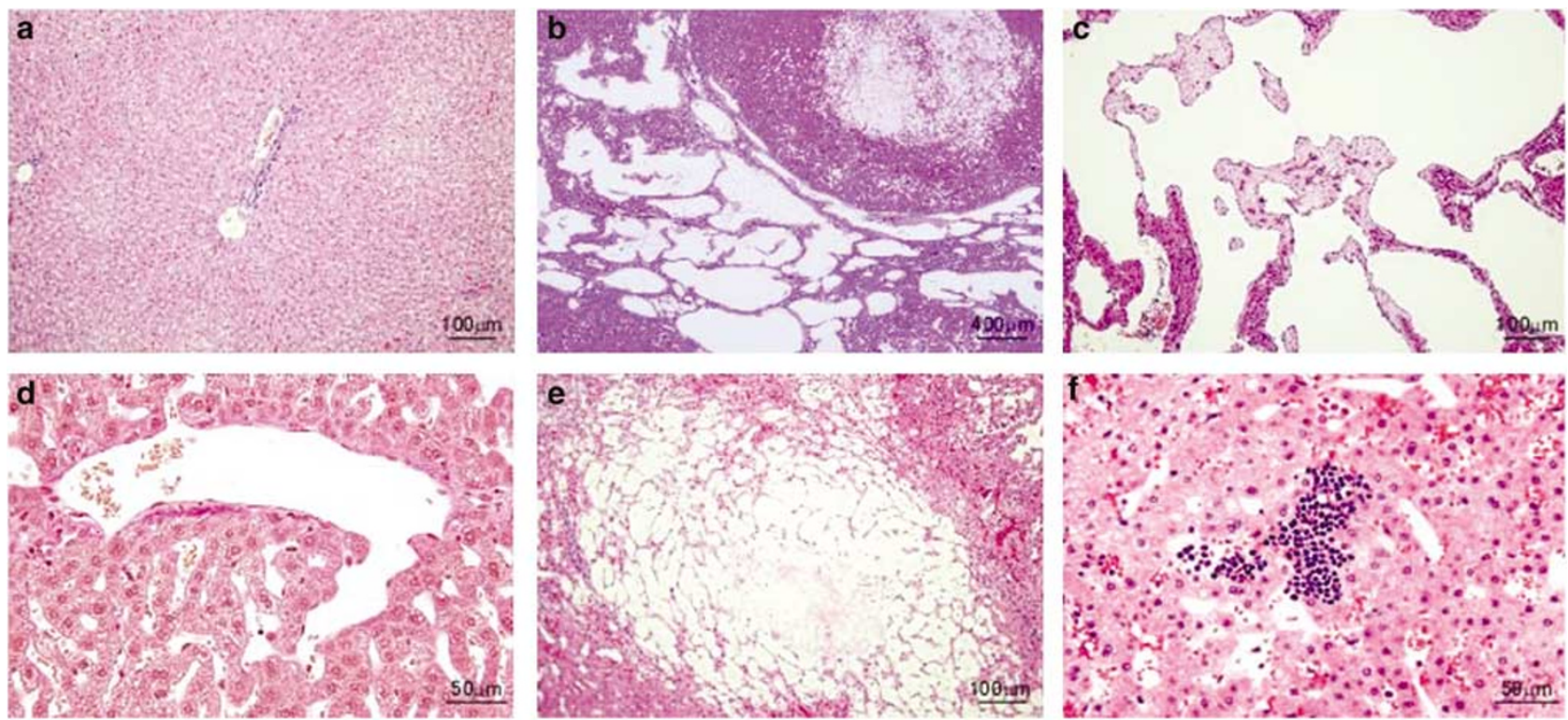

Figure 5 Micrographs of the livers stained by H-E (a-c, e-f) and EVG (d). Control rabbit liver (a). Hemangioma of a Tg rabbit (low magnification) (b). At higher magnification, vascular stroma was clearly seen (c) and many sinusoids were dilated and connected with the central veins (d). Hemorrhage and dropout hepatocytes (e) were frequently observed and embedded in amorphorous materials. Extramedullary hematopoiesis in the sinusoidal space of the liver was noted (f).

these cells are of EC lineage. Beneath these ECs, spindle cells were positive for $\alpha$-SMA, a marker for SMCs and pericytes. This finding is also consistent with the observations in Tg mice with conditional expression of VEGF. ${ }^{28}$ The lumens of these blood vessels were often hyperemic, suggesting that these neo-vessels are connected to the circulation. Taken together, these pathological findings indicated that these malformed vascular structures were those of angiomatosis or diffuse hemangiomas. ${ }^{26}$

A noteworthy finding in VEGF $\mathrm{Tg}$ rabbits was the presence of hematological abnormalities and splenomegaly. Tg rabbits showed decreased numbers of erythrocytes and platelets and had lower levels of plasma iron compared to control rabbits. Examination of the bone marrow revealed that Tg bone marrow showed marked hypercellularity with an increased number of megakaryocytes. In addition, there was prominent extramedullary hematopoiesis in the liver and spleen. Thus, these findings suggest that $\mathrm{Tg}$ rabbits suffered from thrombocytopenia and microcytic hypochromic anemia. Examinations of blood smear samples revealed many deformed or fragmented erythrocytes, indicating that the hemolytic process did indeed occur in $\mathrm{Tg}$ rabbits. This observation was initially surprising and unexpected because in humans, anemia is frequently associated with high levels of VEGF in the plasma. ${ }^{16}$ However, it is generally believed that elevation of VEGF is secondary to anemia or hypoxia as opposed to directly causing anemia. Nevertheless, the occurrence of large hemangiomas is frequently associated with thrombocytopenia, hemolytic anemia and splenomegaly (called Kasabach-Merritt syndrome) in human beings. ${ }^{29-31}$ Thus, our Tg rabbits may represent a new model not only for hepatic diffuse hemangiomas but also for Kasabach-Merritt syndrome.

The anemia in Tg rabbits may have resulted from several possible mechanisms such as sequestration and entrapment of a large amount of RBCs in the hemangiomas or bleeding following rupture of the vascular channels within the hemangiomas. This notion was supported by the observations of severe hyperemia in the liver and the presence of erythrocyte fragmentation in the peripheral blood, indicating that these abnormal vessel channels may lead to mechanical damage of the blood cells. Several theories have been proposed to explain the relationship between the hemangiomas and the thrombocytopenia in Kasabach-Merritt syndrome, ${ }^{32}$ including (1) trapping or utilization of platelets in the hemangioma, (2) increased peripheral destruction of platelets and (3) decreased production of platelets in the bone marrow. In Tg rabbits, the major apparent cause of thrombocytopenia is the 'trapping' effect, because there was a large amount of blood in the hemangiomas, increased number of megakaryocytes in the bone marrow and decreased blood platelet counts. This notion was supported by the observation that the surgical removal of the liver lesions dramatically normalized the levels of platelets in $\mathrm{Tg}$ rabbits (Figure 8).

We also considered the possibility that these rabbits may suffer from microangiopathic hemolysis, although we did not find any fibrin thrombi arising in the abnormal blood vessels. Recently, it has been reported that increased hepatic VEGF may lead to anemia by inhibiting erythropoietin 

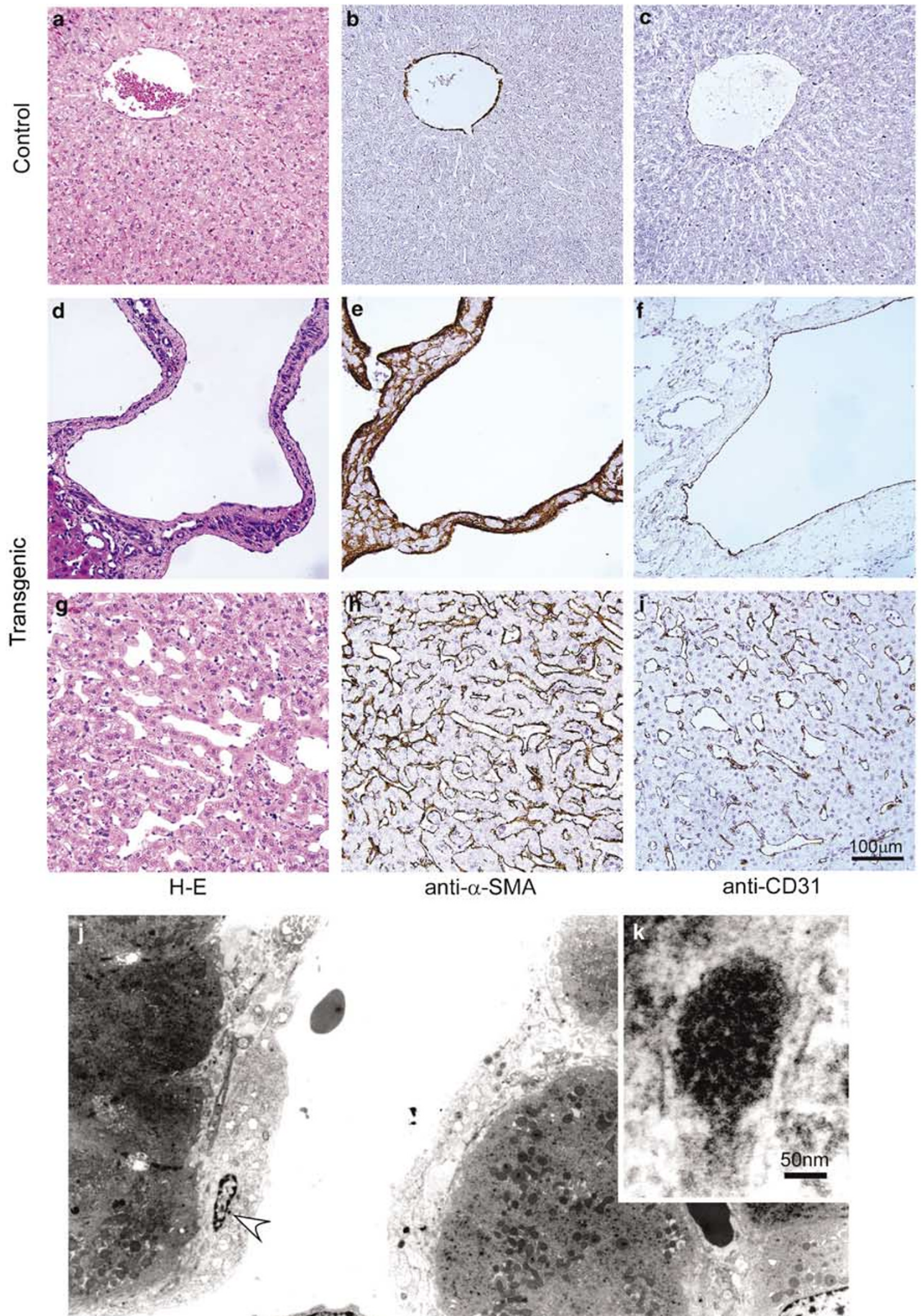

(1)
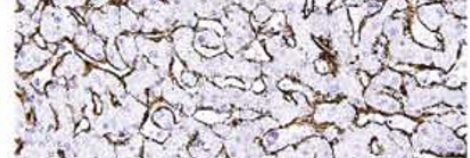

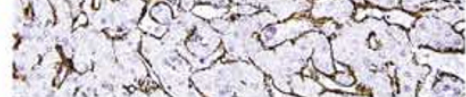
anti- $\alpha$-SMA

anti-CD31

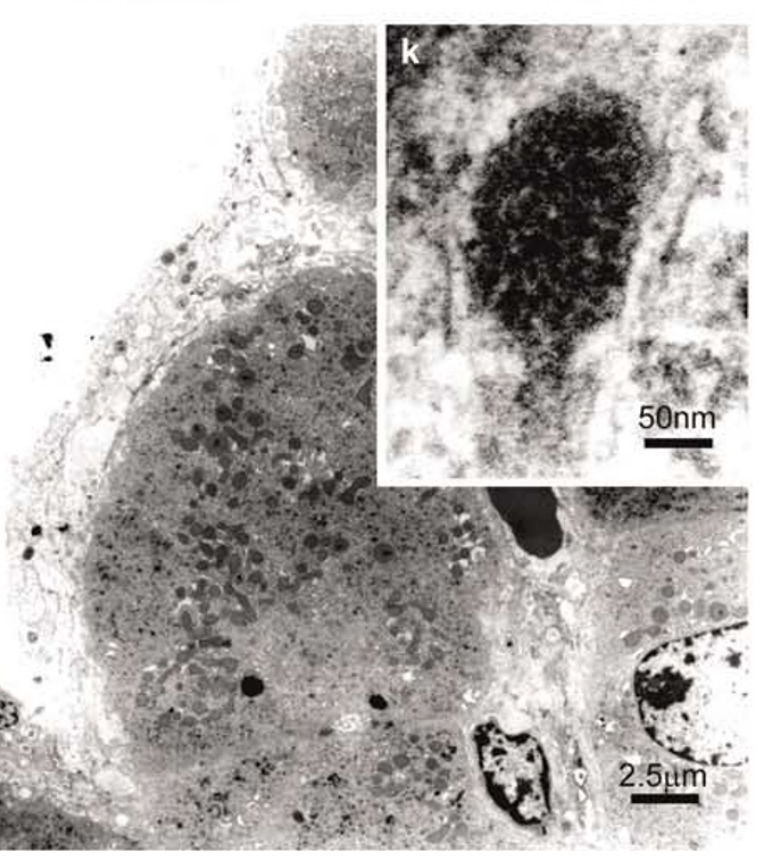


synthesis in the liver independent of the hypoxia pathway. ${ }^{33}$ It may be interesting to investigate whether it also occurs in Tg rabbits in future.

Kasabach-Merritt syndrome is frequently seen in infants and several therapeutic procedures have been used for the treatment, including surgical removal, radiation, corticosteroids or cytotoxic drugs, and recombinant interferon. ${ }^{34,35}$ Two murine models with induced vascular tumors have been reported. In these models, mice were transplanted with either hemangioendotheliomas ${ }^{32}$ or Tg ECs expressing the entire polyoma virus. ${ }^{36}$ Both types of
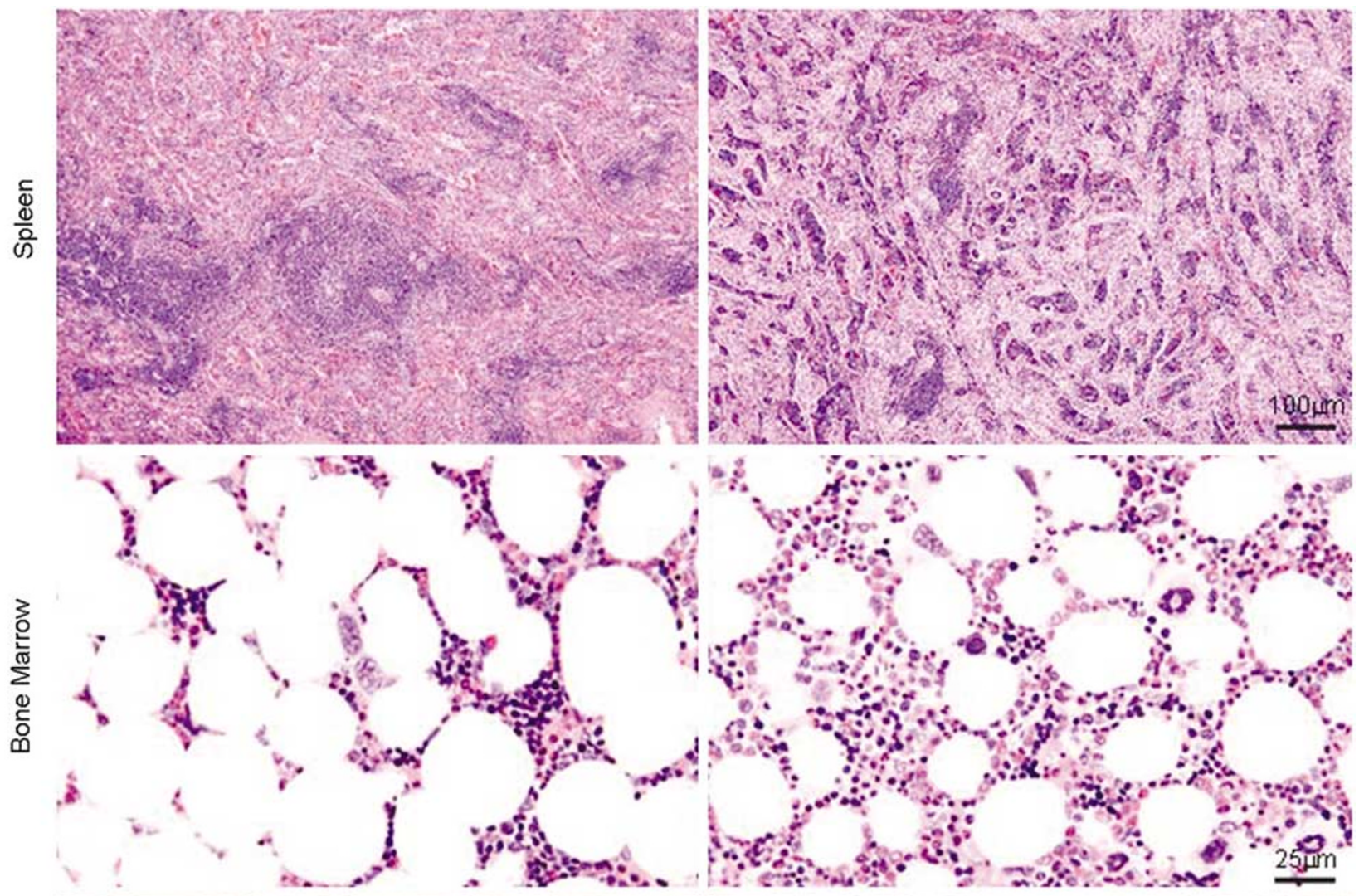

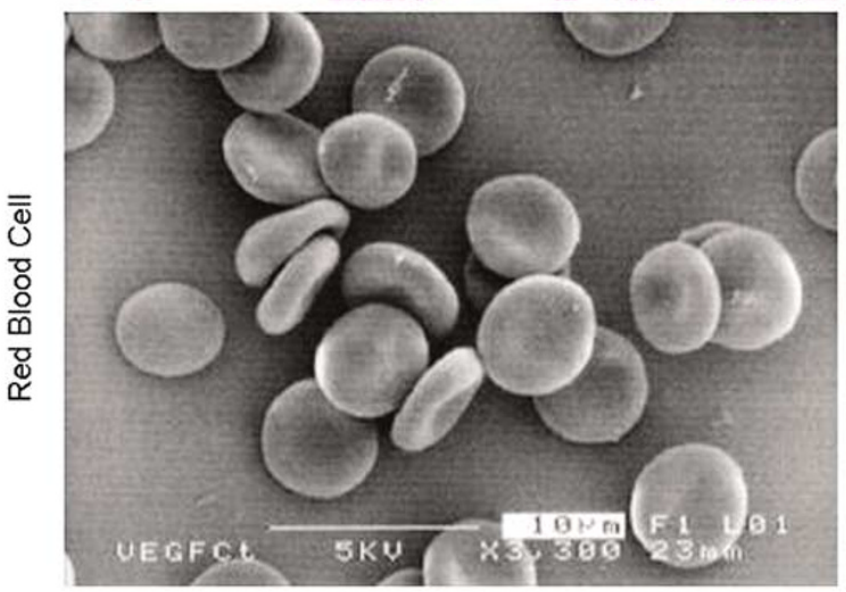

Control

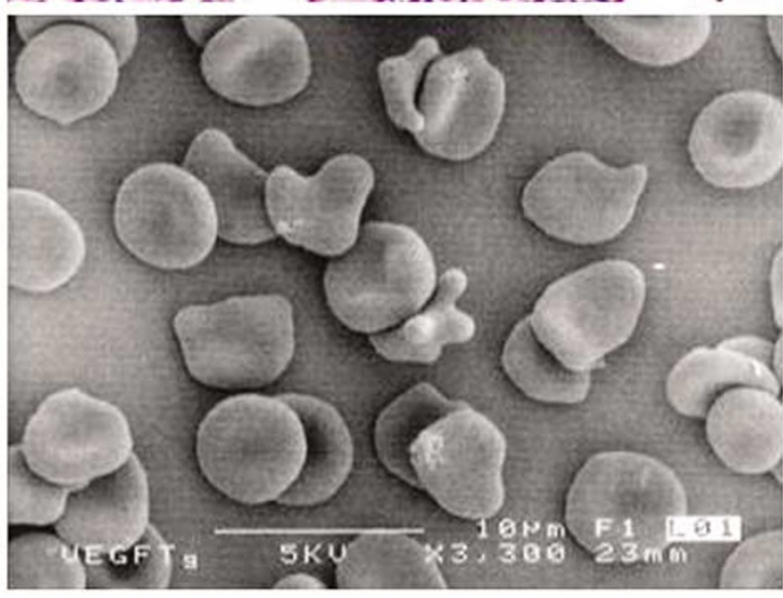

Transgenic

Figure 7 Morphological observation of the spleen (upper), bone marrow (middle) and RBCs (bottom panel). Tg rabbit spleen showed marked extramedullary hematopoiesis whereas their bone marrow was hypercellular. RBCs were examined under a scanning electron microscope (bottom). Note that there are many deformed or fragmented erythrocytes in Tg rabbit blood.

Figure 6 Immunohistochemical and electron microscopic analysis of the liver. (a-c) Control liver, (d-i) Tg rabbit liver, H-E stain(a, d, g), anti $\alpha$-SMA $(\mathbf{b}, \mathbf{e}, \mathbf{h})$, and anti-CD31 (c, f, i). (j-k) Electron microscopic observation of the Weibel-Palade bodies in ECs of hemangiomas. 


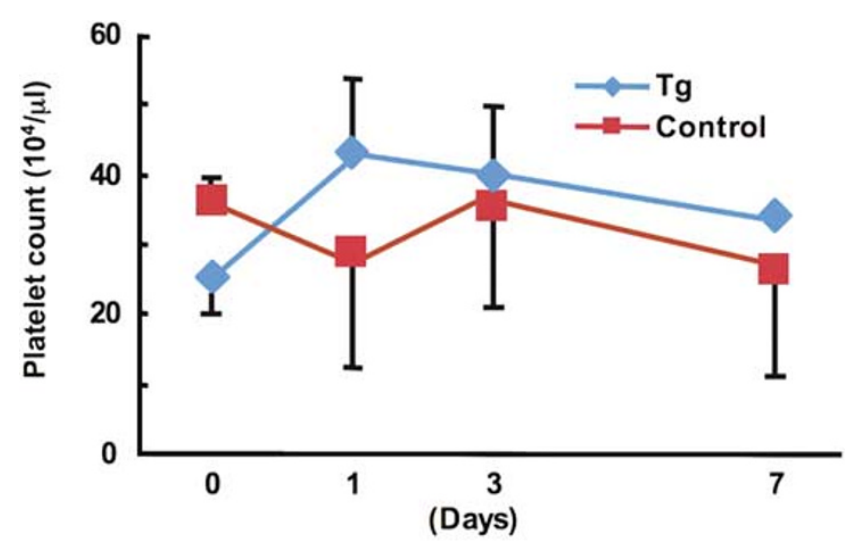

\section{Acknowledgements}

We thank Drs K Nakagawa (Kyushu University School of Medicine) for providing hVEGF ${ }_{165} \mathrm{cDNA}$ plasmid, H Itoh (Kyoto University School of Medicine) for his encouragement of the current study, X Wang, J Liang, for their participation in the study. This work was supported in parts by grantsin-aid for scientific research by Ministry of Education, Culture, Sports and Technology (MEXT), Japan (KAKENHI.16390089 and 16659099, JF), Mitsubishi Pharma Research Foundation, the Takeda Science Foundation, Ono Medical Research Foundation, Novartis Foundation for the Promotion of Science, and a grant of the Center for Tsukuba Advanced

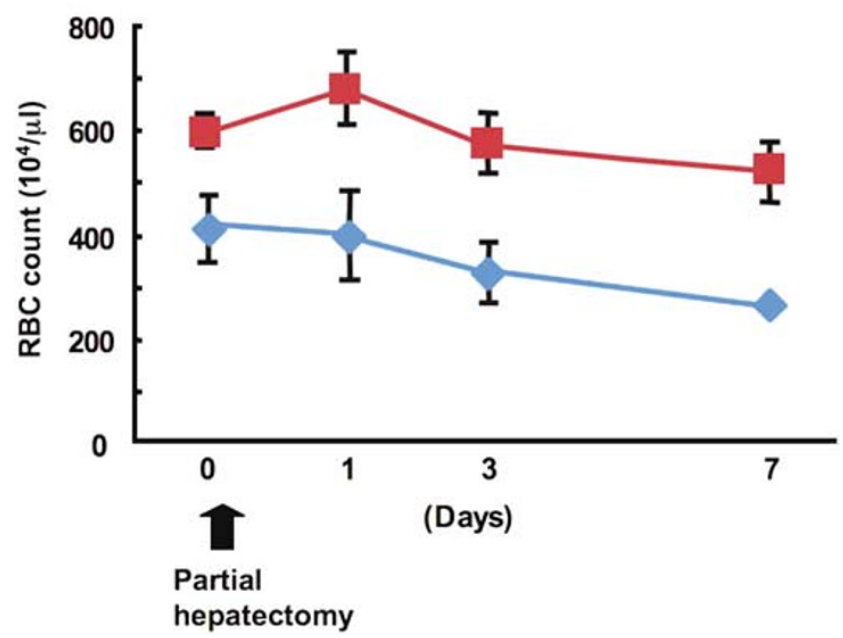

Figure 8 Effect of surgical removal of the hepatic lesions on the platelets and RBCs. Tg and control rabbits $(n=7$ for Tg and 4 for control at age of 26-44 weeks) were subjected to the hepatic surgery as described in Materials and methods and their blood platelets and RBCs were monitored.

mice died within a few weeks after the formation of the tumors. Apparently, the current Tg rabbit model has advantages over mice because the $\mathrm{Tg}$ rabbits survive as long as 2 years and have germline transmission of the phenotypes. Therefore, they are potentially useful for the development and evaluation of therapeutic efficacy of treatments such as surgical intervention (as shown in this study) and angiogenesis inhibitors for KasabachMerritt syndrome or hepatic hemangiomas. ${ }^{37}$

In summary, this study demonstrated that overexpression of VEGF in the liver is sufficient to induce hemangiomas. Hemangiomas accompanied by anemia and thrombocytopenia (which can be corrected through surgical removal of the hepatic lesions) in Tg rabbits with high hepatic expression of VEGF indicate that these Tg rabbits exhibit many features of Kasabach-Merritt syndrome in humans. They provide a new system not only to investigate the pathogenesis of hemangiomas but also to test the effects of purported angiogenesis inhibitors. Future studies will explore the utility of our Tg rabbits in these regards. Tsukuba (JF).

\section{References}

1 Ferrara N. Vascular endothelial growth factor: basic science and clinical progress. Endocr Rev 2004;25: 581-611.

2 Park JE, Keller GA, Ferrara N. The vascular endothelial growth factor (VEGF) isoforms: differential deposition into the subepithelial extracellular matrix and bioactivity of extracellular matrix-bound VEGF. Mol Biol Cell 1993;4:1317-1326.

3 Ferrara N, Carver-Moore K, Chen H, et al. Heterozygous embryonic lethality induced by targeted inactivation of the VEGF gene. Nature 1996;380:439-442.

4 Carmeliet P, Ferreira V, Breier G, et al. Abnormal blood vessel development and lethality in embryos lacking a single VEGF allele. Nature 1996;380:435-439.

5 Folkman J. Tumor angiogenesis and tissue factor. Nat Med 1996;2:167-168.

6 Ohnishi S, Miyagishima T, Nakagawa M, et al. Diffuse neonatal hemangiomatosis without cutaneous lesions in an adult-a case report. Angiology 2002;53:235-237.

7 Caldwell RB, Bartoli M, Behzadian MA, et al. Vascular endothelial growth factor and diabetic retinopathy: pathophysiological mechanisms and treatment perspectives. Diabetes Metab Res Rev 2003;19:442-455.

8 Schrijvers BF, Flyvbjerg A, De Vriese AS. The role of vascular endothelial growth factor (VEGF) in renal pathophysiology. Kidney Int 2004;65:2003-2017.

$9 \mathrm{Kim} \mathrm{NH}$, Oh JH, Seo JA, et al. Vascular endothelial growth factor (VEGF) and soluble VEGF receptor FLT-1 in diabetic nephropathy. Kidney Int 2005;67:167-177.

10 Chen YX, Nakashima Y, Tanaka K, et al. Immunohistochemical expression of vascular endothelial growth factor/vascular permeability factor in atherosclerotic intimas of human coronary arteries. Arterioscler Thromb Vasc Biol 1999;19:131-139.

11 Nakagawa K, Chen YX, Ishibashi H, et al. Angiogenesis and its regulation: roles of vascular endothelial cell growth factor. Semin Thromb Hemost 2000;26:61-66.

12 Celletti FL, Waugh JM, Amabile PG, et al. Vascular endothelial growth factor enhances atherosclerotic plaque progression. Nat Med 2001;7:425-429.

13 Ballara S, Taylor PC, Reusch P, et al. Raised serum vascular endothelial growth factor levels are associated with destructive change in inflammatory arthritis. Arthritis Rheum 2001;44:2055-2064. 
14 Kim KJ, Li B, Winer J, et al. Inhibition of vascular endothelial growth factor-induced angiogenesis suppresses tumour growth in vivo. Nature 1993;362: 841-844.

15 Okamoto N, Tobe T, Hackett SF, et al. Transgenic mice with increased expression of vascular endothelial growth factor in the retina: a new model of intraretinal and subretinal neovascularization. Am J Pathol 1997; 151:281-291.

16 Dunst J, Becker A, Lautenschlager C, et al. Anemia and elevated systemic levels of vascular endothelial growth factor (VEGF). Strahlenther Onkol 2002;178: 436-441.

17 Fan J, Watanabe T. Transgenic rabbits as therapeutic protein bioreactors and human disease models. Pharmacol Ther 2003;99:261-282.

18 Kitajima S, Morimoto M, Liu E, et al. Overexpression of lipoprotein lipase improves insulin resistance induced by a high-fat diet in transgenic rabbits. Diabetologia 2004;47:1202-1209.

19 Wang X, Liang J, Koike $\mathrm{T}$, et al. Overexpression of human matrix metalloproteinase-12 enhances the development of inflammatory arthritis in transgenic rabbits. Am J Pathol 2004;165:1375-1383.

20 Houck KA, Leung DW, Rowland AM, et al. Dual regulation of vascular endothelial growth factor bioavailability by genetic and proteolytic mechanisms. J Biol Chem 1992;267:26031-26037.

21 Garcia I, Miyazaki Y, Araki K, et al. Transgenic mice expressing high levels of soluble TNF-R1 fusion protein are protected from lethal septic shock and cerebral malaria, and are highly sensitive to Listeria monocytogenes and Leishmania major infections. Eur J Immunol 1995;25:2401-2407.

22 Fan J, Wang J, Bensadoun A, et al. Overexpression of hepatic lipase in transgenic rabbits leads to a marked reduction of plasma high density lipoproteins and intermediate density lipoproteins. Proc Natl Acad Sci USA 1994;91:8724-8728.

23 Unoki H, Fan J, Watanabe T. Low-density lipoproteins modulate endothelial cells to secrete endothelin-1 in a polarized pattern: a study using a culture model system simulating arterial intima. Cell Tissue Res 1999;295:89-99.

24 Koike T, Liang J, Wang X, et al. Overexpression of lipoprotein lipase in transgenic Watanabe heritable hyperlipidemic rabbits improves hyperlipidemia and obesity. J Biol Chem 2004;279:7521-7529.

25 Yamaoka Y, Ohsawa T, Takasan H, et al. Energy requirement in regenerative and atrophic processes of the liver in man and other mammals. Surg Gynecol Obstet 1974;139:234-240.

26 Weiss S, Goldblum J. Enzinger and Weiss's Soft Tissue Tumors, 4th edn. Mosby: St Louis, MO, 2001.

27 Chang J, Most D, Bresnick S, et al. Proliferative hemangiomas: analysis of cytokine gene expression and angiogenesis. Plast Reconstr Surg 1999;103:1-9; discussion 10.

28 Dor Y, Djonov V, Abramovitch R, et al. Conditional switching of VEGF provides new insights into adult neovascularization and pro-angiogenic therapy. EMBO J 2002;21:1939-1947.

29 Kasabach H, Merritt K. Capillary hemangioma with extensive purpura. Am J Dis Child 1940;59:1063-1070.

30 Sutherland DA, Clark H. Hemangioma associated with thrombocytopenia. Report of a case and review of the literature. Am J Med 1962;33:150-157.

31 Shim WK. Hemangiomas of infancy complicated by thrombocytopenia. Am J Surg 1968;116:896-906.

32 Hoak JC, Warner ED, Cheng HF, et al. Hemangioma with thrombocytopenia and microangiopathic anemia (Kasabach-Merrittsyndrome): an animal model. J Lab Clin Med 1971;77:941-950.

33 Tam BYY, Hoffman J, Park S, et al. Hypoxia-independent regulation of hepatic erythropoietin production by vascular endothelial growth factor. Blood 2004;104: Abstract 2163.

34 Warrell Jr RP, Kempin SJ, Benua RS, et al. Intratumoral consumption of indium-111 labeled platelets in a patient with hemangiomatosis and intravascular coagulation (Kasabach-Merrittsyndrome). Cancer 1983; 52:2256-2260.

35 Larsen EC, Zinkham WH, Eggleston JC, et al. syndrome: therapeutic considerations. Pediatrics 1987;79: 971-980.

36 Dubois-Stringfellow N, Kolpack-Martindale L, Bautch $\mathrm{VL}$, et al. Mice with hemangiomas induced by transgenic endothelial cells. A model for the KasabachMerrittsyndrome. Am J Pathol 1994;144:796-806.

37 Boon LM, Burrows PE, Paltiel HJ, et al. Hepatic vascular anomalies in infancy: a twenty-seven-year experience. J Pediatr 1996;129:346-354. 